\title{
Influence of sea otters on soft-bottom prey communities in southeast Alaska
}

\author{
Rikk G. Kvitek, John S. Oliver \\ Moss Landing Marine Laboratories, PO Box 450, Moss Landing, California 95039, USA
}

\begin{abstract}
Sea otters Enhydra lutris, well documented as 'keystone' predators in rocky marine communities, were found to exert a strong influence on infaunal prey communities in soft-sediment habitats. The effect of sea otter predation in subtidal soft-bottom prey communities in southeast Alaska was evaluated via a natural experiment comparing prey populations in similar habitats with and without sea otters. Results indicate that otters forage primarily on bivalve prey, especially the butter clam Saxidomus giganteus and dramatically reduce infaunal bivalve and sea urchin (Strongylocentrotus spp.) prey populations. Bivalve prey abundance, biomass and size were negatively correlated with sea otter occupancy. Otter-cracked shells of the deep-burrowing clams Tresus capax and Panope abrupta were rarely found, even at otter foraging sites where these clams accounted for the majority of available prey biomass, suggesting that these species have a partial depth refuge from otter predation. The differential impacts of otter predation on prey populations suggest that infaunal bivalves provide a more sustainable food base than do sea urchins.
\end{abstract}

\section{INTRODUCTION}

Sea otters Enhydra lutris are known to forage extensively in both rocky (VanBlaricom \& Estes 1988) and soft-bottom (Kvitek \& Oliver 1988, Kvitek 1990, Kvitek et al. 1992) habitats throughout their northeast Pacific range. The ability of sea otters to greatly reduce prey abundance and consequently alter community structure in rocky habitats is well documented (Estes \& Palmisano 1974, Dayton 1975, Estes et al. 1978, Simenstad et al. 1978, Duggins 1980, Kvitek et al. 1989), and has led to the characterization of the sea otter as a classic keystone (sensu Paine 1969) predator (Levin 1988, but see Foster \& Schiel 1988). In these habitats sea otter predation limits the size and abundance of large, herbivorous invertebrates (e.g. sea urchins and abalone) which leads to a proliferation of kelps and associated fauna (Estes et al. 1978, VanBlaricom \& Estes 1988).

In contrast to rocky habitats, relatively little is known regarding the ability of sea otters to limit prey populations in soft-bottom communities (Kvitek \& Oliver 1988). The few studies that did directly sample softbottom prey populations have not shown a significant impact of sea otters on prey populations (Hines \& Loughlin 1980, Kvitek et al. 1988). These results, how- ever, may very likely be due to the very limited spatial and temporal scopes of the studies (Kvitek \& Oliver 1988). The excellent studies linking the collapse of the Pismo clam fishery along California beaches to an expanding sea otter population, while very convincing within their scope, are limited by a narrow taxonomic focus and reliance on catch data rather than a comprehensive census of the available prey (Miller et al. 1975, Stephenson 1977, Wendell et al. 1986). There is only one study that has documented the impact of extended sea otter predation ( $\geq 20 \mathrm{yr}$ ) on soft-bottom prey communities across a geographically vast area (the Kodiak archipelago) (Kvitek et al. 1992). By sampling prey populations and shell remains left by feeding sea otters along a gradient of otter occupancy, Kvitek et al. (1992) utilized a natural experiment to correlate a decline in the abundance and size of infaunal prey and sea urchins with length of sea otter occupancy. Because the Kodiak study is the only one of its kind, and because the prey and otter gradients were correlated with a latitudinal gradient in that study, we chose to undertake the research reported here to test further the hypothesis that sea otters control prey populations in soft-bottom communities as they have been shown to do in rocky habitats. 


\section{A natural experiment}

Otters are still recolonizing historical feeding grounds following their near extermination during the fur trade of the 18th and 19th centuries (Kenyon 1969). While this recolonization provides a unique opportunity to explore the effects of otters on prey populations, communities and habitats, the recovery process has not been well documented for either rocky or softsediment environments. There are, however, numerous examples of differences in prey populations and communities in rocky habitats with and without sea otters (Estes \& Paimisano 1974, Dayton 1975, Estes et al. 1978, Simenstad et al. 1978, Duggins 1980, Duggins et al. 1989, Kvitek et al. 1989), but only one from softsediment environments (Kvitek et al. 1992).

Once abundant in southeast Alaska, sea otters had been eliminated from that part of their range before 1900 as a result of the fur trade during the 18th and 19th centuries (Kenyon 1969). Between 1965 and 1968, a total of 402 sea otters were translocated from Amchitka Island (357) and Prince William Sound, Alaska (45) into 6 areas of southeast Alaska (Fig. 1) (Jameson et al. 1982). In 1987, a comprehensive boat survey showed the sea otter population to have increased to 3600 , and that otters had re-colonized nearly half their original range in that region during the preceding 20 yr (Pitcher 1987). This pattern of sea otter expansion into historically occupied areas provided the opportunity to test the impact of sea otters on their prey communities based on a natural experiment. Using the results of the Pitcher (1987) survey, we were able to select 'otter-free' control or reference sites to be compared with sites occupied by otters. Because otters had been reintroduced at 6 isolated sites along the entire length of southeast Alaska, we were able to intersperse otter and non-otter study sites so as to eliminate the potentially confounding effect of latitude.

As in the Kodiak study, our focus was to correlate differences in the abundance and size structure of prey populations in soft-bottom habitats (infaunal bivalves and green sea urchins Strongylocentrotus droebachiensis) with differences in sea otter occupancy. (Although Strongylocentrotus spp. are generally considered to prefer rocky habitats, the green sea urchin is a common and abundant inhabitant of soft-bottom communities throughout much of southern Alaska; Kvitek 1990, Kvitek et al. 1992). A secondary objective was to determine the importance of the butter clam Saxidomus giganteus as a food resource for sea otters, and the impact of sea otter predation on butter clam populations in southeast Alaska. Butter clams have been shown to remain chronically toxic throughout much of the inside passage of southeast Alaska as a result of sequestered, highly lethal paralytic shellfish poisoning toxins (PST) (see Nishitani \& Chew 1988 for review). It has also been argued that butter clams have evolved to utilized these sequestered toxins as a chemical defense (Kvitek 1991, Kvitek \& Beitler 1991. Kvitek et al. 1991, Kvitek 1992). Because sea otters have been shown to shun butter clams containing PST, it has been hypothesized that otters may avoid some butter clam populations or entire regions of southeast Alaska dominated by toxic butter clams (Kvitek et al. 1991). Therefore, the extent to which toxic dinoflagellate blooms (the source of PST) may mediate sea otter predation and distribution will vary with the importance of butter clams in the sea otter diet and the distribution and dominance of butter clams in southeast Alaska.

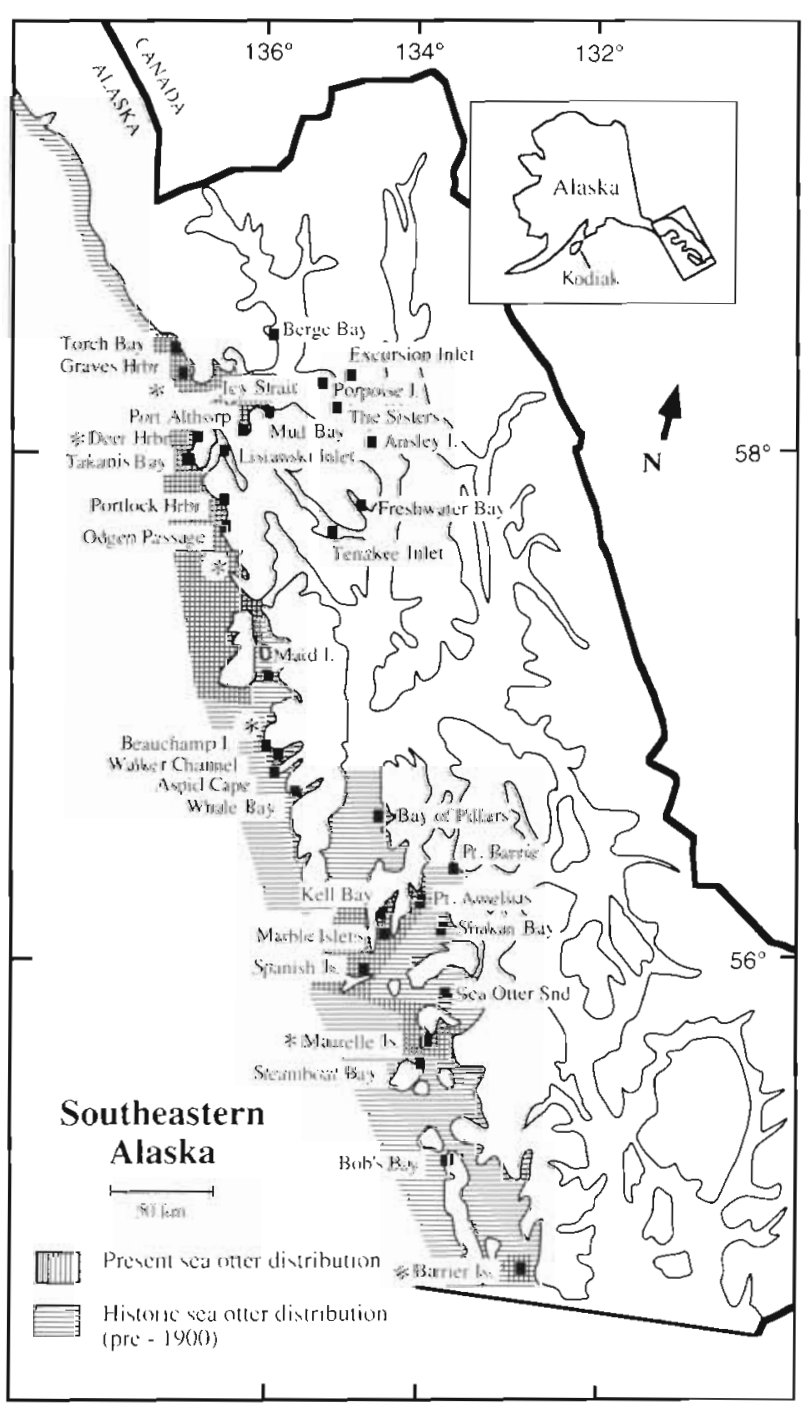

Fig. 1 Southeastern Alaska showing the locations of the 33 study areas, the present and historic range of sea otters and the 6 areas $(*$ ) where translocated otters were reintroduced into the region between 1965 and 1968 


\section{METHODS}

Benthic prey populations and bivalve shell remains were sampled at a total of 46 sites within the 33 study areas shown in Fig. 1. All work was done between March 1988 and August 1990 from aboard the RV 'Alpha Helix'. Prey sampling techniques used in this study are the same as those employed by Kvitek et al. (1992) in the Kodiak research.

Study site selection. Study areas within the historic sea otter range were divided into 2 levels of nested categories. The first level was whether an area had been subject to sea otter predation or not (Table 1). 'No otters' areas were those: where no otters or very few sea otters ( 2 in the case of Lisianski Inlet) had been observed during the Pitcher (1987) survey or by our field crews; where bivalve shells discarded by feeding otters (otter-cracked shells) were absent or very rare; and where red sea urchins Strongylocentrotus franciscanus were either abundant or large. Such urchin populations rarely persist in Alaska in the presence of sea otters (for review see VanBlairicom \& Estes 1988 , but see Foster \& Schiel 1988). 'Otter' areas were those where abundant sea otters had been observed during the 1987 census and where otter-cracked bivalve shells were generally common.

The second level of site classification was by type of bivalve assemblage present. The majority of bivalve biomass in most of the infaunal communities sampled was contributed by either butter clams or Humilaria kennerlyi (another venerid clam of comparable size). For this reason, sites were grouped according to whether or not butter clams accounted for $>50 \%$ of bivalve biomass.

In addition to the areas described above, 6 areas were chosen as representative of bivalve communities within the inside passage of southeast Alaska, and therefore potentially outside the historic range of sea otters (Fig. 1) (Kvitek et al. 1991).

More than one specific study site was chosen within some study areas (Table 1). This was done if examples of more than one type of prey assemblage were found in an area, and in the case of larger study areas. All sites were subtidal between the depths of 3 and $20 \mathrm{~m}$ and represent the richest bivalve habitats that could be found by divers within each area.

Shell record. Divers collected bivalve shells haphazardly from the sea floor at each site (except Barrier Is. 1 \& 2). Shells were sorted by species and whether or not sea otter predation was the probable cause of death (Kvitek et al. 1992). All shells were measured to the nearest $\mathrm{mm}$.

Live prey. Strongylocentrotus franciscanus and $S$. droebachiensis (hereafter red and green sea urchins respectively) abundance and size were sampled either at the soft-bottom site or an adjacent rocky outcrop.
Urchins were sampled at the depth of their greatest abundance at each site. Haphazardly placed transects $(1 \times 10 \mathrm{~m}$ or $2 \times 10 \mathrm{~m})$ were used to determine abundance. The smaller area transect was used when urchins were too numerous to count on a single dive using the larger sampling area. In areas occupied by sea otters but not areas where sea otters were absent, all rocks $\leq 1 \mathrm{~m}$ encountered along the transect were turned over and inspected for sea urchins. Mean size for both species was determined by measuring the test diameter of every urchin encountered in haphazardly tossed quadrats $\left(1 \mathrm{~m}^{2}\right)$. At the Maurelle Is. site, we recorded whether rocks ( 30 to $100 \mathrm{~cm}$ across) encountered along the urchin transect had been recently turned over. A rock, with its upper surface covered by vividly colored or starkly white invertebrates, stands out clearly in the presence of other rocks covered with the drab and dull, brown kelps and red algae characteristic of the region. This was taken as an indication of rocks having been flipped by foraging otters. It is unlikely that the displacement of these rocks was caused by wave action at this site given the depth of $20 \mathrm{~m}$. The percentage of rocks flipped was not recorded at 'No otter' sites, which were sampled before otter-occupied sites, because, at the time, this was an unanticipated result, and had not been incorporated into the sampling design.

Density, biomass and sizes of infaunal bivalves $\geq 30$ $\mathrm{mm}$ were obtained for all sites by excavating haphazardly placed $0.25 \mathrm{~m}^{2}$ cylinders to a depth of $50 \mathrm{~cm}$ with a diver-held water pump (see Kvitek et al. 1992). Biomass was determined from drained wet meat weights. Shell lengths were measured to the nearest mm. At 2 sites in the Barrier Islands, geoduck (Panope abrupta) abundance was sampled by counting their large and conspicuous bifurcate siphons along transects rather than excavation due to the great burrow depth of this species. This method gives a minimum estimate of abundance, because only those individuals with their siphons exposed at the time are counted

\section{RESULTS}

\section{Test of study site classification}

Bivalve shells cracked by sea otters were rarely found at sites outside the established sea otter range (mean $=2 \%$ of all shells collected) but were significantly more common at sites occupied by sea otters (mean $=33 \%$ of all shells collected) $(t$-test, $\mathrm{df}=27$, $t=6.6, \mathrm{p}=0.0001$, percentages arcsine-transformed) (Table 1). Red sea urchins, where sampled, were significantly more abundant ( $t$-test, $\mathrm{df}=13, t=2.4$, $\mathrm{p}=0.02$ ) and larger ( $t$-test, $\mathrm{df}=13, t=7.7, \mathrm{p}=0.0001$ ) at sites without sea otters than at sites where otters were common (Table 1). 
Table 1. Indicators of sea otter occupancy and predation level used in study site classification. Otter counts are from a 1987 Alaska Department of Fish and Game sea otter census (Pitcher 1987). Shells cracked by sea otters is the percentage of total shells collected at a site showing signs of sea otter predation. Abundance and test diameter of red Strongylocentrotus franciscanus and green $S$. droebachiensis sea urchins were also used as indicators of otter predation level. All sea urchins found at sites occupied by sea otters were located under rocks, with the exception of urchins at Mud Bay, and $40 \%$ and $25 \%$ of the red and green urchins respectively at the Maurelle Is. site. Sample size $(\mathrm{N})$ for urchin abundance is the number of $1 \times 10 \mathrm{~m}$ or $2 \times 10 \mathrm{~m}$ transects used in counting the urchins. Absent = never observed; present $=$ observed but not sampled

\begin{tabular}{|c|c|c|c|c|c|c|c|c|c|c|c|}
\hline \multirow[t]{3}{*}{ Study site } & \multirow{3}{*}{$\begin{array}{c}1987 \\
\text { otter } \\
\text { count }\end{array}$} & \multirow{2}{*}{\multicolumn{2}{|c|}{$\begin{array}{l}\text { Shells cracked } \\
\text { by sea otters }\end{array}$}} & \multicolumn{4}{|c|}{ S. franciscanus } & \multicolumn{4}{|c|}{ S. droebachiensis } \\
\hline & & & & \multicolumn{2}{|c|}{ Abundance } & \multicolumn{2}{|c|}{ Test diameter } & \multicolumn{2}{|c|}{ Abundance } & \multicolumn{2}{|c|}{ Test diameter } \\
\hline & & $(\%)$ & $N$ & (ind. $\mathrm{m}^{-2}$ ) & $N$ & $(\mathrm{~mm})$ & $N$ & (ind. $\mathrm{m}^{-2}$ ) & $N$ & $(\mathrm{~mm})$ & $N$ \\
\hline \multicolumn{12}{|l|}{ 'Non-otter' sites } \\
\hline Aspid Cape & 0 & Absent & & Present & & $70 \pm 24$ & 68 & Present & & & \\
\hline Bay of Pillars 1 & 0 & 0 & 215 & $6.4 \pm 2.4$ & 9 & $115 \pm 51$ & 57 & Present & & & \\
\hline Bay of Pillars 2 & 0 & 0 & 72 & Present & & Present & & Present & & & \\
\hline Beauchamp Is. & 0 & 8 & 166 & $10.4 \pm 4.1$ & 4 & $78 \pm 32$ & 22 & $0.0 \pm 0.0$ & 4 & & \\
\hline Bob's Bay & 0 & Absent & & $40.0 \pm 1.7$ & 3 & $82 \pm 41$ & 21 & $2.6 \pm 0.4$ & 3 & & \\
\hline Lisianski 1 & 2 & 0 & 232 & Present & & & & Present & & & \\
\hline Lisianski, Mìner Is. & 2 & 0 & 212 & Present & & & & Present & & & \\
\hline Maid Is. & 0 & 0 & 185 & $4.4 \pm 1.4$ & 6 & $96 \pm 26$ & 96 & Present & & & \\
\hline Pt. Barrie 1 & 0 & Absent & & Absent & & & & Present & & & \\
\hline Pt. Barrie 2 & 0 & Absent & & Absent & & & & $37.6 \pm 21.9$ & 10 & $22 \pm 6$ & 79 \\
\hline Pt. Barrie 3 & 0 & Absent & & Absent & & & & $13.3 \pm 8.1$ & 4 & $60 \pm 7$ & 50 \\
\hline Sea Otter Sound 1 & 0 & 0 & 453 & Present & & $91 \pm 20$ & 39 & Present & & & \\
\hline Sea Otter Sound 2 & 0 & Absent & & Present & & & & Present & & & \\
\hline Shakan Bay & 0 & 5 & 148 & $5.4 \pm 2.4$ & 3 & $89 \pm 23$ & 44 & Present & & & \\
\hline Steamboat Bay & 0 & 1 & 266 & Present & & $94 \pm 30$ & 40 & Present & & & \\
\hline Walker Channel & 0 & 3 & 79 & $5.4 \pm 1.0$ & 5 & $75 \pm 19$ & 72 & $0.8 \pm 1.1$ & 5 & $31 \pm 15$ & 72 \\
\hline Whale Bay & 0 & 1 & 180 & $6.5 \pm 0.1$ & 3 & $84 \pm 38$ & 41 & $<0.1$ & 3 & & \\
\hline Grand mean $\pm \mathrm{SD}$ & $0 \pm 1$ & $2 \pm 3$ & 11 & $11.2 \pm 12.8$ & 7 & $87 \pm 13$ & 10 & $9.1 \pm 16.2$ & 6 & $37 \pm 16$ & 3 \\
\hline \multicolumn{12}{|l|}{ Otter sites } \\
\hline Afflect Canal & 229 & & & & & & & & & & \\
\hline Kell Bay & & 11 & 193 & $0.0 \pm 0.0$ & 3 & & & $0.0 \pm 0.0$ & 3 & & \\
\hline Kell Bay central & & 11 & 195 & $0.0 \pm 0.0$ & 3 & & & $0.0 \pm 0.0$ & 3 & & \\
\hline Kell Bay W. & & 37 & 399 & $0.0 \pm 0.0$ & 3 & & & $0.0 \pm 0.0$ & 3 & & \\
\hline Marble Islets & & 39 & 258 & $0.0 \pm 0.0$ & 3 & & & $0.0 \pm 0.0$ & 3 & & \\
\hline Barrier Islands & 147 & & & & & & & & & & \\
\hline Barrier Is. 1 & & Present & & $0.1 \pm 0.1$ & 3 & $52 \pm 10$ & 5 & $0.2 \pm 0.2$ & 3 & $36 \pm 10$ & 12 \\
\hline Barrier Is. 2 & & Present & & Absent & & & & Absent & & & \\
\hline Barrier Is. 3 & & 39 & 239 & Absent & & & & Absent & & & \\
\hline Barrier Is. 4 & & 23 & 502 & Absent & & & & Absent & & & \\
\hline Deer Harbor & 57 & 5 & 257 & $1.3 \pm 1.2$ & 3 & $36 \pm 12$ & 51 & $0.7 \pm 0.5$ & 3 & $23 \pm 7$ & 40 \\
\hline Graves Harbor & 89 & 61 & 399 & Absent & & & & Absent & & & \\
\hline Maurelle Is. & 312 & 11 & 338 & $0.1 \pm 0.1$ & 3 & $29 \pm 6$ & 5 & $0.2 \pm 0.1$ & 3 & $28 \pm 7$ & 14 \\
\hline Mud Bay & 75 & 58 & 500 & Absent & & & & $187.5 \pm 36.3$ & 22 & $18 \pm 6$ & 91 \\
\hline Odgen Passage & 40 & 26 & 686 & Absent & & & & Absent & & & \\
\hline Port Althorp N. & 192 & 44 & 391 & Absent & & & & Absent & & & \\
\hline Port Althorp W. & 192 & 68 & 298 & Absent & & & & Absent & & & \\
\hline Portlock Harbor & 81 & 34 & 177 & Present & & $32 \pm 15$ & 34 & Present & & $29 \pm 6$ & 17 \\
\hline Pt. Amelius & 15 & 30 & 76 & $0.2 \pm 0.1$ & 2 & $16 \pm 5$ & 4 & $3.1 \pm 1.7$ & 2 & $21 \pm 5$ & 62 \\
\hline Spanish Is. & 92 & 60 & 343 & Absent & & & & Absent & & & \\
\hline Takanis Bay & 56 & 2 & 334 & Absent & & & & Absent & & & \\
\hline Torch Bay & 28 & 37 & 523 & Absent & & & & Absent & & & \\
\hline Grand mean $\pm S D$ & $115 \pm 87$ & $33 \pm 20$ & 18 & $0.2 \pm 0.4$ & 8 & $33 \pm 13$ & 5 & $21 \pm 62$ & 9 & $26 \pm 7$ & 6 \\
\hline
\end{tabular}

\section{Shell record}

On average, 36,21 and $20 \%$ of the shells of butter clams, Humilaria kennerlyi and remaining bivalve species respectively, which were collected at sites occu- pied by sea otters, showed signs of sea otter predation (Table 2). These values are minimum estimates of bivalve mortality due to sea otters because many shells, especially older ones, were not able to be assigned to a mortality source unambiguously (Kvitek et al. 1992). 
Table 2. Minimum proportion of bivalve mortality attributable to sea otter predation as inferred from incidence of shell damage in collections at sites occupied by sea otters. These values are minimum estimates of otter-induced mortality because many shells, especially older ones, were not able to be assigned unambiguously to a mortality source. 'Butter clam' sites are those at which butter clams Saxidomus giganteus make up $>50 \%$ of the bivalve biomass (Tables $3 \& 4$ ). Only those sites at which total shell collections (otter cracked plus all other shells) were made are shown

\begin{tabular}{|c|c|c|c|c|c|c|}
\hline \multirow[t]{2}{*}{ Study site } & \multicolumn{2}{|c|}{ Butter clams } & \multicolumn{2}{|c|}{ Humilaria kennerlyi } & \multicolumn{2}{|c|}{ Other spp. } \\
\hline & Otter-cracked & $\mathrm{N}$ & Otter-cracked & N & Otter-cracked & $\mathrm{N}$ \\
\hline \multicolumn{7}{|l|}{ 'Butter clam' sites } \\
\hline Barrier Is 3 & 38 & 133 & & 0 & 41 & 106 \\
\hline Kell Bay & 13 & 158 & 0 & 16 & 5 & 19 \\
\hline Kell Bay central & 17 & 110 & 0 & 38 & 4 & 47 \\
\hline Kell W. & 47 & 304 & & 0 & 5 & 95 \\
\hline Marble Islets & 42 & 217 & & 0 & 22 & 41 \\
\hline Mud Bay & 69 & 354 & & 0 & 31 & 146 \\
\hline Ogden Passage & 30 & 562 & 18 & 11 & 9 & 113 \\
\hline Port Althorp N. & 66 & 222 & & 0 & 14 & 169 \\
\hline Portlock Harbor & 38 & 142 & & 0 & 17 & 35 \\
\hline Torch Bay 2 & 46 & 366 & 0 & 2 & 17 & 155 \\
\hline Grand mean $\pm \mathrm{SD}$ & $41 \pm 18$ & 10 & $5 \pm 9$ & 4 & $17 \pm 12$ & 10 \\
\hline \multicolumn{7}{|c|}{ Bivalve assemblages not dominated by butter clams } \\
\hline Barrier Is. 4 & 15 & 78 & 36 & 145 & 18 & 279 \\
\hline Deer Harbor 2 & 1 & 149 & 21 & 29 & 6 & 79 \\
\hline Graves Harbor & 68 & 286 & 33 & 15 & 46 & 98 \\
\hline Maurelle & 17 & 12 & 13 & 179 & 7 & 147 \\
\hline Port Althorp W. & 80 & 246 & 0 & 7 & 13 & 45 \\
\hline Pt. Amelius & 9 & 11 & 37 & 49 & 25 & 16 \\
\hline Spanish Is. & 28 & 46 & 59 & 122 & 70 & 175 \\
\hline Takanis Bay & 0 & 42 & 100 & 1 & 2 & 291 \\
\hline Grand mean $\pm \mathrm{SD}$ & $27 \pm 30$ & 8 & $37 \pm 31$ & 8 & $23 \pm 23$ & 8 \\
\hline
\end{tabular}

\section{Live prey}

Green urchins were observed at all non-otter sites, they were found at only $30 \%$ of the sites occupied by sea otters, and all urchins found at otter sites were located under rocks, with the exception of those at Mud Bay and at Maurelle Is. (Table 1). Green urchins did not differ significantly either in abundance ( $t$-test, $\mathrm{df}=13, t=0.47, \mathrm{p}=0.33$ ) or size ( $t$-test, $\mathrm{df}=7, t=1.4$, $p=0.10)$ between the otter and non-otter areas where they were sampled. However, because rocks at sites without otters were not turned over and searched by divers, the actual abundances at those sites may be greater than reported here. Additionally, although the high abundance of green urchins at Mud Bay was an exception to the general pattern and primarily responsible for the high $\mathrm{p}$ value in the $t$-test above $(1$ tail $t$-test, $\mathrm{df}=12, t=1.67, \mathrm{p}=0.06$, with Mud Bay data excluded), Mud Bay also had the smallest urchins of any site (Table 1), with all urchins being less than $3 \mathrm{~cm}$, and thus of little value to otters as prey.

At Maurelle Is., the site where sea urchins were most frequently observed being eaten by sea otters $(33 \%$ of the observed prey items taken by feeding otters; Kvitek et al. unpubl.), $56 \%$ of the rocks $(\mathrm{N}=50)$ encountered along the urchin transect had been flipped recently, presumable by foraging otters. Although not quantitatively sampled at otter-free sites, we never observed overturned rocks at these sites, and we do not believe such a conspicuous phenomenon could have been overlooked.

Bivalve biomass and abundance (excluding Panope abrupta for reasons given below) were generally much lower at sites occupied by sea otters (Tables $3 \& 4$ ). At sites where butter clams account for $>50 \%$ of the bivalve biomass (Table 3), the wet meat weights of all clams combined and butter clams alone, were significantly lower in otter areas (1-tailed $t$-test, df $=17$, $t=3.2, \mathrm{p}=0.003$, and $t=3.5, \mathrm{p}=0.002$ respectively). Biomass for the remaining species was lower at otter sites but the difference was not significant (1-tailed $t$-test, $\mathrm{df}=17, t=1, \mathrm{p}=0.17$ ). Bivalve densities were not significantly lower in otter areas (1-tailed $t$-test, $\mathrm{df}=17, \mathrm{p}>0.05$ ). However, the lack of significance in the latter 2 tests was due to a single high density area of butter clams and littleneck clams Protothaca staminea that may have been 'unpalatable' to sea otters at the Barrier Is. 3 site. The mantle tissues of these 2 bivalve species were generally dark, leathery and adhering in dry, discolored patches to the shell interior. Many of the shells were also misshapen. We have never encountered clams in this condition anywhere else. 
Table 3. Biomass and abundance (mean $\pm \mathrm{SD}$ ) of live bivalves $\geq 30 \mathrm{~mm}$ collected at sites where butter clams Saxidomus giganteus accounted for $>50 \%$ of the bivalve biomass. Means for all species combined (Total), butter clams only, and remaining species (Other spp.) are given. $\mathrm{N}=$ number of $0.25 \mathrm{~m}^{2}$ quadrats excavated. A single high density but possibly 'unpalatable' butter clams and littleneck clams (Protothaca staminea) community was located at the Barrier Is. 3 otter site (see 'Results')

\begin{tabular}{|c|c|c|c|c|c|c|c|}
\hline \multirow[t]{2}{*}{ Study sites } & \multicolumn{3}{|c|}{ Bivalve prey biomass $\left(g 0.25 \mathrm{~m}^{-2}\right)$} & \multicolumn{3}{|c|}{ Bivalve prey abundance (ind. $0.25 \mathrm{~m}^{-2}$ ) } & \multirow[t]{2}{*}{ s } \\
\hline & Total & Butter clams & Other spp. & Total & Butter clams & Other spp. & \\
\hline \multicolumn{8}{|l|}{ ‘Non-otter' sites } \\
\hline Bay of Pillars 2 & $490 \pm 186$ & $431 \pm 215$ & $59 \pm 61$ & $14.7 \pm 8.0$ & $11.3 \pm 7.9$ & $3.0 \pm 4.0$ & 6 \\
\hline Lisianski 1 & $1037 \pm 381$ & $1035 \pm 379$ & $2 \pm 6$ & $12.4 \pm 2.2$ & $12.3 \pm 2.1$ & $0.0 \pm 0.0$ & 7 \\
\hline Lisianski Miner I. & $1224 \pm 781$ & $1195 \pm 800$ & $29 \pm 19$ & $31.4 \pm 13.6$ & $27.2 \pm 16.6$ & $4.0 \pm 3.0$ & 5 \\
\hline Maid I. & $491 \pm 159$ & $444 \pm 71$ & $47 \pm 29$ & $12.4 \pm 1.5$ & $7.4 \pm 1.1$ & $5.0 \pm 2.0$ & 5 \\
\hline Pt. Barrie 1 & $1662 \pm 470$ & $1621 \pm 467$ & $41 \pm 100$ & $36.2 \pm 10.2$ & $36.0 \pm 10.1$ & $0.0 \pm 0.0$ & 6 \\
\hline Pt. Barrie 2 & $148 \pm 149$ & $148 \pm 149$ & $0 \pm 0$ & $1.7 \pm 1.6$ & $1.7 \pm 1.6$ & $0.0 \pm 0.0$ & 10 \\
\hline Sea Otter Sound 1 & $573 \pm 301$ & $358 \pm 241$ & $215 \pm 85$ & $22.0 \pm 12.0$ & $7.5 \pm 6.7$ & $15.0 \pm 6.0$ & 6 \\
\hline Shakan Bay & $1613 \pm 322$ & $905 \pm 174$ & $708 \pm 315$ & $31.7 \pm 9.0$ & $13.2 \pm 2.9$ & $19.0 \pm 8.0$ & 6 \\
\hline Grand mean $\pm \mathrm{SD}$ & $904 \pm 564$ & $767 \pm 503$ & $137 \pm 240$ & $20.3 \pm 12.0$ & $14.6 \pm 11.4$ & $5.8 \pm 7.3$ & 8 \\
\hline \multicolumn{8}{|l|}{ Otter sites } \\
\hline Barrier Is. 3 & $1420 \pm 588$ & $988 \pm 591$ & $432 \pm 153$ & $115.0 \pm 26.2$ & $44.2 \pm 29.5$ & $71.0 \pm 20.0$ & 5 \\
\hline Kell Bay & $123 \pm 112$ & $119 \pm 114$ & $3 \pm 5$ & $2.3 \pm 1.5$ & $2.0 \pm 1.7$ & $0.0 \pm 1.0$ & 6 \\
\hline Kell Bay central & $49 \pm 54$ & $49 \pm 54$ & $0 \pm 0$ & $0.8 \pm 0.8$ & $0.8 \pm 0.8$ & $0.0 \pm 0.0$ & 5 \\
\hline Kell Bay W. & $190 \pm 93$ & $143 \pm 107$ & $47 \pm 72$ & $15.2 \pm 15.0$ & $6.2 \pm 2.3$ & $9.0 \pm 14.0$ & 6 \\
\hline Marble Islets & $50 \pm 31$ & $33 \pm 30$ & $21 \pm 12$ & $3.3 \pm 1.9$ & $1.3 \pm 1.2$ & $2.0 \pm 1.0$ & 5 \\
\hline Maurelle Is. & $18 \pm 31$ & $14 \pm 32$ & $4 \pm 9$ & $0.4 \pm 0.5$ & $0.2 \pm 0.4$ & $0.0 \pm 0.0$ & 5 \\
\hline Mud Bay & $133 \pm 46$ & $103 \pm 30$ & $30 \pm 19$ & $9.4 \pm 4.2$ & $4.6 \pm 1.3$ & $5.0 \pm 3.0$ & 5 \\
\hline Odgen Passage & $14 \pm 40$ & $14 \pm 40$ & $0 \pm 0$ & $0.1 \pm 0.4$ & $0.1 \pm 0.4$ & $0.0 \pm 0.0$ & 8 \\
\hline Port Althorp N. & $22 \pm 25$ & $14 \pm 19$ & $8 \pm 8$ & $3.8 \pm 3.6$ & $1.6 \pm 1.5$ & $2.0 \pm 2.0$ & 5 \\
\hline Portlock Harbor & $23 \pm 23$ & $22 \pm 22$ & $1 \pm 2$ & $1.8 \pm 1.0$ & $1.7 \pm 0.8$ & $0.0 \pm 0.0$ & 6 \\
\hline Torch Bay 2 & $80 \pm 31$ & $44 \pm 43$ & $36 \pm 26$ & $6.4 \pm 3.6$ & $2.2 \pm 1.6$ & $4.0 \pm 3.0$ & 5 \\
\hline Grand mean $\pm \mathrm{SD}$ & $193 \pm 411$ & $140 \pm 285$ & $53 \pm 127$ & $14.4 \pm 33.7$ & $5.9 \pm 12.8$ & $8.5 \pm 20.9$ & 11 \\
\hline
\end{tabular}

Table 4 . Biomass and abundance (mean \pm SD) of live bivalves $\geq 30 \mathrm{~mm}$ collected at sites where butter clams Saxidomus giganteus accounted for $<50 \%$ of the bivalve wet meat biomass. Means for all species combined (Total), and the species having the highest biomass (Major sp.) are given. $\mathrm{N}=$ number of $0.25 \mathrm{~m}^{2}$ quadrats excavated. Major bivalve species are: Humilaria kennerlyi, Protothaca staminea, butter clam Saxidomus giganteus, and Tresus capax

\begin{tabular}{|c|c|c|c|c|c|c|}
\hline \multirow[t]{2}{*}{ Study sites } & \multicolumn{3}{|c|}{ Bivalve prey biomass ( $90.25 \mathrm{~m}^{-2}$ ) } & \multicolumn{3}{|c|}{ Bivalve prey abundance (ind. $0.25 \mathrm{~m}^{-2}$ ) } \\
\hline & Total & Maj & sp. & Total & Major sp. & $\mathrm{N}$ \\
\hline \multicolumn{7}{|l|}{ 'Non-otter' sites } \\
\hline Aspid Cape & $609 \pm 141$ & H. kennerlyi & $143 \pm 67$ & $23.2 \pm 7.5$ & $9.0 \pm 3.5$ & 6 \\
\hline Bay of Pillars 1 & $515 \pm 155$ & H. kennerlyi & $344 \pm 133$ & $19.0 \pm 3.6$ & $14.8 \pm 6.2$ & 9 \\
\hline Beauchamp Is. & $181 \pm 108$ & H. kennerlyi & $398 \pm 119$ & $11.7 \pm 5.4$ & $16.4 \pm 7.0$ & 6 \\
\hline Bob's Bay & $177 \pm 47$ & H. kennerlyi & $177 \pm 47$ & $11.5 \pm 1.7$ & $11.5 \pm 1.7$ & 4 \\
\hline Pt. Barrie 3 & $207 \pm 60$ & H. kennerlyj & $181 \pm 44$ & $8.8 \pm 2.1$ & $8.0 \pm 1.9$ & 6 \\
\hline Sea Otter Sound 2 & $461 \pm 224$ & F. kennerlyi & $123 \pm 37$ & $11.0 \pm 3.0$ & $4.0 \pm 1.7$ & 6 \\
\hline Steamboat Bay & $2029 \pm 1185$ & $T$ capax & $1387 \pm 1033$ & $64.2 \pm 41.6$ & $20.8 \pm 21.0$ & 6 \\
\hline Walker Channel & $388 \pm 155$ & H. kennerlyi & $152 \pm 92$ & $14.8 \pm 2.1$ & $5.0 \pm 2.8$ & 6 \\
\hline Whale Bay & $1338 \pm 250$ & H. kennerlyi & $744 \pm 238$ & $42.0 \pm 1.1 .0$ & $34.3 \pm 10.0$ & 6 \\
\hline Grand mean $\pm \mathrm{SD}$ & $656 \pm 626$ & & $405 \pm 418$ & $22.9 \pm 18.5$ & $13.8 \pm 9.4$ & 9 \\
\hline \multicolumn{7}{|l|}{ Otter sites } \\
\hline Barrier Is. 1 & $14 \pm 12$ & H. kennerlyi & $12 \pm 8$ & $1.8 \pm 1.5$ & $1.5 \pm 1.0$ & 6 \\
\hline Barrier Is. 2 & $291 \pm 121$ & H. kennerlyi & $245 \pm 96$ & $15.2 \pm 6.4$ & $11.8 \pm 4.8$ & 6 \\
\hline Barrier Is 4 & $112 \pm 103$ & H. kennerlyi & $47 \pm 41$ & $7.5 \pm 6.9$ & $3.7 \pm 3.8$ & 6 \\
\hline Deer Harbor & $57 \pm 50$ & $P$. staminea & $12 \pm 6$ & $6.2 \pm 2.9$ & $2.2 \pm 1.2$ & 6 \\
\hline Graves Harbor & $330 \pm 361$ & T. capax & $239 \pm 381$ & $3.7 \pm 2.1$ & $1.4 \pm 1.8$ & 9 \\
\hline Port Althorp W. & $80 \pm 76$ & Butter clam & $28 \pm 19$ & $11.0 \pm 8.6$ & $3.4 \pm 1.8$ & 5 \\
\hline Pt. Amelius & $19 \pm 13$ & H. kennerlyi & $18 \pm 14$ & $2.2 \pm 1.3$ & $2.0 \pm 1.0$ & 5 \\
\hline Spanish Is. & $32 \pm 17$ & H. kennerlyi & $22 \pm 17$ & $7.5 \pm 4.4$ & $5.5 \pm 4.2$ & 6 \\
\hline Takanis Bay & $17 \pm 13$ & P. staminea & $17 \pm 14$ & $2.8 \pm 1.9$ & $2.8 \pm 1.9$ & 5 \\
\hline Torch Bay 1 & $44 \pm 29$ & Butter clam & $12 \pm 15$ & $3.4 \pm 1.1$ & $0.6 \pm 0.5$ & 5 \\
\hline Grand mean $\pm S D$ & $100 \pm 116$ & & $65 \pm 94$ & $6.1 \pm 4.3$ & $3.5 \pm 3.2$ & 10 \\
\hline
\end{tabular}


At sites where the butter clam accounted for $<50 \%$ of the bivalve biomass, the same pattern of abundance held true for all bivalves combined, and for bivalve species contributing the most biomass at a given site (Table 4). Biomass and abundance were significantly lower where otters were common (1-tail t-tests, $p \leq 0.05$ ). Bivalve biomass sampled at the 6 sites along the inside passage of southeast Alaska, and outside the sea otter range, was also high and overwhelmingly dominated by the butter clam (Table 5).

Shell lengths of live bivalves were significantly smaller at sites occupied by sea otters. At sites where butter clams accounted for $>50 \%$ of the bivalve biomass, butter clams $(1$-tailed $t$-test, $\mathrm{df}=17, t=3.6$, $p=0.001$ ) and the pooled individuals of all other bivalve species present (1-tailed $t$-test, $\mathrm{df}=12, t=$ 2.3, $p=0.02$ ) were smaller at otter sites (Table 6). The same pattern was found when comparing bivalve sizes from sites where butter clams make up <50\% of the bivalve biomass, both for butter clams (1-tailed $t$-test, $\mathrm{df}=10, t=2.8, \mathrm{p}=0.01$ ) and for the pooled individuals of all other bivalves species present (1-tailed $t$-test, df $=19, t=2.0, p=0.03)$ (Table 7). Sites with live shell length sample sizes of $<2$ were omitted from these analyses.

Panope abrupta (the largest and deepest burrowing clam in the northeast Pacific ${ }_{i}$ Morris et al. 1980) were encountered at 6 sites, and although the sample size is not sufficient for statistical analysis, the values for biomass, abundance and size do not vary greatly between otter and non-otter areas (Table 8). In fact, $P$. abrupta biomass at sites within the otter range was comparable to the biomass values for all other species combined at sites without sea otters (Tables $3 \& 4$ ).

Table 5. Bivalve biomass at sites along the inside passage of Southeast Alaska, and outside the reported historic range of sea otters. All bivalve species excavated from $0.25 \mathrm{~m}^{2}$ quadrats were combined for the total wet meat biomass (mean $\pm \mathrm{SD}$ ). The percent of total biomass contributed by butter clams Saxidomus giganteus is also given. $N=$ number of quadrats excavated

\begin{tabular}{|lccc|}
\hline Study site & $\begin{array}{c}\text { Total biomass } \\
\left(\mathrm{g} 0.25 \mathrm{~m}^{-2}\right)\end{array}$ & $\begin{array}{c}\text { Percent } \\
\text { butter clams }\end{array}$ & $\mathrm{N}$ \\
\hline Ansley Is. & $541 \pm 277$ & 97 & 5 \\
Berge Bay & $1823 \pm 604$ & 88 & 3 \\
Excursion Inlet 1 & $687 \pm 406$ & 68 & 4 \\
Excursion Inlet 2 & $390 \pm 182$ & 82 & 5 \\
Freshwater Bay & $442 \pm 238$ & 93 & 5 \\
Porpoise Is. & $426 \pm 274$ & 81 & 5 \\
Tenakee Inlet & $1277 \pm 175$ & 78 & 6 \\
The Sisters 1 & $316 \pm 196$ & 61 & 5 \\
The Sisters 2 & $400 \pm 251$ & 32 & 5 \\
Grand mean $\pm \mathrm{SD}$ & $700 \pm 512$ & $77 \pm 21$ & 9 \\
\hline
\end{tabular}

Table 6 . Shell lengths (mean $\pm S D$ ) of live bivalves $\geq 30 \mathrm{~mm}$ collected at sites where butter clams Saxodomus giganteus dccounted for the majority of the bivalve biomass. Sites with samples sizes of 1 were omitted

\begin{tabular}{|c|c|c|c|c|}
\hline \multirow[t]{2}{*}{ Study site } & \multicolumn{4}{|c|}{ Live bivalve prey size ( $\mathrm{mm}$ ) } \\
\hline & Butter clams & $N$ & Other bivalves & $\mathrm{N}$ \\
\hline \multicolumn{5}{|l|}{ 'Non-otter' sites } \\
\hline Bay of Pillars 2 & $68 \pm 21$ & 68 & $60 \pm 16$ & 20 \\
\hline Lisianski 1 & $96 \pm 16$ & 86 & 55 & 1 \\
\hline Lisianski Miner Is. & $76: \pm 13$ & 136 & $42 \pm 7$ & 21 \\
\hline Maid I. & $86 \pm 6$ & 37 & $43 \pm 15$ & 25 \\
\hline Pt. Barrie 1 & $76 \pm 12$ & 216 & 125 & 1 \\
\hline Pt. Barrie 2 & $99 \pm 5$ & 17 & & \\
\hline Sea Otter Sound 1 & $74 \pm 23$ & 45 & $54 \pm 13$ & 88 \\
\hline Shakan Bay & $86 \pm 22$ & 79 & $59 \pm 26$ & 111 \\
\hline Grand mean $\pm S D$ & $84 \pm 12$ & 8 & $63 \pm 28$ & 7 \\
\hline \multicolumn{5}{|l|}{ Otter sites } \\
\hline Barrier Is. 3 & $59 \pm 11$ & 221 & $38 \pm 7$ & 354 \\
\hline Kell Bay & $83 \pm 18$ & 12 & $47 \pm 1$ & 2 \\
\hline Kell Bay central & $80 \pm 29$ & 4 & & \\
\hline Kell Bay W. & $57 \pm 17$ & 37 & $37 \pm 6$ & 54 \\
\hline Marble Islets & $62 \pm 6$ & 8 & $44 \pm 7$ & 12 \\
\hline Mud Bay & $59 \pm 9$ & 23 & $41 \pm 5$ & 24 \\
\hline Port Althorp N. & $42 \pm 10$ & 8 & $31 \pm 2$ & 11 \\
\hline Portlock Harbor & $47 \pm 14$ & 10 & 35 & 1 \\
\hline Torch Bay 2 & $55 \pm 16$ & 11 & $45 \pm 12$ & 21 \\
\hline Grand mean $\pm S D$ & $60 \pm 14$ & 9 & $40 \pm 5$ & 8 \\
\hline
\end{tabular}

Table 7 Shell lengths (mean \pm SD) of live bivalves $\geq 30 \mathrm{~mm}$ collected at sites where butter clams Saxodomus giganteus accounted for $<50 \%$ of the bivalve biomass. Sites with samples sizes of 1 were omitted from the table. $N=$ number of individuals

\begin{tabular}{|c|c|c|c|c|}
\hline \multirow{2}{*}{ Study site } & \multicolumn{4}{|c|}{ Live bivalve prey size (mm) } \\
\hline & Butter clams & $N$ & Other bivalves & $s \mathrm{~N}$ \\
\hline \multicolumn{5}{|l|}{ 'Non-otter' sites } \\
\hline Aspid Cape & & & $72 \pm 11$ & 116 \\
\hline Bay of Pillars 1 & $87 \pm 24$ & 11 & $69 \pm 14$ & 160 \\
\hline Beauchamp I. & $56 \pm 25$ & 5 & $57 \pm 15$ & 65 \\
\hline Bob's Bay & & & $58 \pm 16$ & 46 \\
\hline Pt. Barrie 3 & $82 \pm 3$ & 2 & $70 \pm 8$ & 51 \\
\hline Sea Otter Sound 2 & $82 \pm 27$ & 19 & $70 \pm 23$ & 44 \\
\hline Steamboat Bay & $55 \pm 24$ & 66 & $58 \pm 22$ & 319 \\
\hline Walker Channel & $107 \pm 3$ & 4 & $70 \pm 14$ & 85 \\
\hline Whale Bay & $101 \pm 10$ & 34 & $68 \pm 14$ & 219 \\
\hline Grand mean $\pm \mathrm{SD}$ & $81 \pm 20$ & 7 & $66 \pm 6$ & 9 \\
\hline \multicolumn{5}{|l|}{ Otter sites } \\
\hline Barrier Is. 1 & & & $46 \pm 9$ & 11 \\
\hline Barrier Is. 2 & & & $65 \pm 13$ & 91 \\
\hline Barrier Is. 4 & $40 \pm 10$ & 2 & $58 \pm 14$ & 43 \\
\hline Deer Harbor & $47 \pm 10$ & 5 & $42 \pm 11$ & 32 \\
\hline Graves Harbor & $75 \pm 23$ & 19 & $101 \pm 21$ & 14 \\
\hline Port Althorp W. & $41 \pm 7$ & 17 & $38 \pm 10$ & 38 \\
\hline Pt. Amelius & & & $46 \pm 10$ & 11 \\
\hline Spanish Is. & & & $36 \pm 5$ & 45 \\
\hline Takanis Bay & & & $39 \pm 4$ & 14 \\
\hline Torch Bay 1 & $56 \pm 16$ & 3 & $49 \pm 15$ & 14 \\
\hline Grand mean $\pm \mathrm{SD}$ & $52 \pm 14$ & 5 & $52 \pm 20$ & 10 \\
\hline
\end{tabular}


Table 8. Panopea abrupta (geoduck). Biomass, abundance and shell length at sites with and without sea otters

\begin{tabular}{|c|c|c|c|c|c|c|c|}
\hline \multirow[t]{2}{*}{ Study sites } & \multicolumn{2}{|l|}{ Biomass } & \multicolumn{2}{|l|}{ Abundance } & \multirow{2}{*}{$\begin{array}{l}\text { Sampling unit } \\
\text { area }\left(m^{2}\right)\end{array}$} & \multicolumn{2}{|c|}{ Size } \\
\hline & $\left(g 0.25 \mathrm{~m}^{-2}\right)$ & $N$ & (ind. $0.25 \mathrm{~m}^{-2}$ ) & $N$ & & $(\mathrm{~mm})$ & $N$ \\
\hline \multicolumn{8}{|l|}{ 'Non-otter' sites } \\
\hline Sea Otter Sound & $1785 \pm 1282$ & 6 & $1.8 \pm 1.2$ & 6 & 0.25 & $150 \pm 17$ & 11 \\
\hline Steamboat Bay & $2290 \pm 1574$ & 6 & $3.3 \pm 2.0$ & 6 & 0.25 & $124 \pm 40$ & 20 \\
\hline \multicolumn{8}{|l|}{ Otter sites } \\
\hline Barrier Is. 5 & $1856 \pm 655$ & 6 & $2.8 \pm 1.0^{\mathrm{a}}$ & 29 & 0.25 & $132 \pm 9$ & 17 \\
\hline Barrier Is. 4 & Not sampled & & $1.8 \pm 1.0^{\mathrm{a}}$ & 7 & 10 & Not sampled & \\
\hline Deer Harbor & $449 \pm 518$ & 6 & $0.5 \pm 0.5$ & 6 & 0.25 & $147 \pm 15$ & 3 \\
\hline Maurelle Is. & $953 \pm 905$ & 5 & $1.6 \pm 1.1$ & 5 & 0.25 & $140 \pm 12$ & 6 \\
\hline
\end{tabular}

In summary, taken as a whole, the otter cracked shell record from southeast Alaska was numerically dominated by butter clams (Table 9). Compared with other species, butter clams were generally more numerous in the otter-cracked record than in the live bivalve assemblage (Table 9).

\section{DISCUSSION}

\section{Effects of sea otter predation}

As in Kodiak (Kvitek et al. 1992), the expanding sea otter populations in southeast Alaska provided an opportunity to correlate differences in soft-bottom prey communities with the presence or absence of sea otters. Now that 36 sites within and 33 sites outside sea otter ranges in Kodiak and southeast Alaska have been surveyed, a general pattern of prey exploitation and impact is emerging. Dense populations of large bivalves or sea urchins do not persist in the presence of sea otters. Prey size, abundance and biomass were, with few exceptions, much lower in areas occupied by sea otters (Tables 1, 3, 4, 5, 6 \& 7). These differences in prey populations are most probably a result of the high mortality due to predation by otters, which is clearly reflected in the shell record (Table 2).

Only 2 bivalve prey species, Tresus capax and Panope abrupta, the largest and deepest burrowing clam species encountered, appeared to have a relative refuge from predation by sea otters. Large

Table 9. Frequency occurrence of butter clams Saxodomus giganteus among sea otter-cracked shells (dead) and live bivalves at each site. Occurrence in the otter-cracked and live bivalve assemblage is also given for the species that was the most or next most common after butter clams in the otter-cracked shell collection. $\mathrm{N}=$ total number of individuals collected for all species. Other bivalve species include: Clinocardium spp., Gari sp., Humilaria kennerlyi, Macoma spp., Mya spp., Protothaca staminea, Spisula polynima and Tresus capax

\begin{tabular}{|c|c|c|c|c|c|c|c|}
\hline \multirow[t]{2}{*}{ Study site } & \multicolumn{2}{|c|}{ Butter clams } & \multicolumn{3}{|c|}{ Other most common species among otter-cracked shells } & \multicolumn{2}{|c|}{$\mathrm{N}$} \\
\hline & Otter-cracked & Live & Species & Otter-cracked & Live & Dead & Live \\
\hline Barrier Is. 1 & $3 \%$ & $0 \%$ & H. kennerlyi & $49 \%$ & $82 \%$ & 105 & 11 \\
\hline Barrier Is. 3 & $55 \%$ & $38 \%$ & P. staminea & $27 \%$ & $57 \%$ & 216 & 575 \\
\hline Barrier Is. 4 & $9 \%$ & $4 \%$ & H. kennerlyi & $42 \%$ & $49 \%$ & 335 & 45 \\
\hline Deer Harbor & $21 \%$ & $14 \%$ & Garisp. & $40 \%$ & $14 \%$ & 80 & 37 \\
\hline Graves Harbor & $81 \%$ & $58 \%$ & T. capax & $9 \%$ & $33 \%$ & 650 & 33 \\
\hline Kell Bay & $85 \%$ & $86 \%$ & P. staminea & $9 \%$ & $7 \%$ & 99 & 14 \\
\hline Kell Bay central & $96 \%$ & $100 \%$ & P. staminea \& Mya spp. & $4 \%$ & $0 \%$ & 95 & 4 \\
\hline Kell Bay W. & $92 \%$ & $41 \%$ & P. staminea & $7 \%$ & $56 \%$ & 357 & 91 \\
\hline Marble Islets & $93 \%$ & $40 \%$ & P. staminea & $7 \%$ & $10 \%$ & 161 & 20 \\
\hline Mud Bay & $85 \%$ & $51 \%$ & P. staminea & $15 \%$ & $49 \%$ & 298 & 47 \\
\hline Ogden Passage & $86 \%$ & $100 \%$ & P. staminea & $8 \%$ & $0 \%$ & 563 & 1 \\
\hline Port Althorp N. & $96 \%$ & $42 \%$ & Clinocardium spp. & $2 \%$ & $0 \%$ & 133 & 19 \\
\hline Port Althorp W. & $97 \%$ & $31 \%$ & Macoma spp. & $3 \%$ & $20 \%$ & 204 & 55 \\
\hline Portlock Harbor & $93 \%$ & $91 \%$ & P. staminea & $6 \%$ & $0 \%$ & 335 & 11 \\
\hline Pt. Amelius & $5 \%$ & $0 \%$ & H. kennerlyi & $82 \%$ & $91 \%$ & 22 & 11 \\
\hline Spanish I. & $8 \%$ & $0 \%$ & H. kennerlyi & $53 \%$ & $73 \%$ & 223 & 45 \\
\hline Takanis Bay & $32 \%$ & $0 \%$ & P. staminea & $53 \%$ & $100 \%$ & 57 & 14 \\
\hline Torch Bay 2 & $87 \%$ & $34 \%$ & 5. polynima & $4 \%$ & $0 \%$ & 539 & 32 \\
\hline
\end{tabular}


T. capax could still be found in the presence of sea otters at Graves Harbor (Table 4) and at Kodiak sites (Kvitek et al. 1992). These large clams were generally found at twice the $20 \mathrm{~cm}$ burrow depth of butter clams and at times in excess of $50 \mathrm{~cm}$. The great burrow depth of $P$. abrupta (ca $1 \mathrm{~m}$ ), the largest of all bivalves in the north Pacific (Morris et al. 1980), may also account for the persistence of this species within the sea otter range (Table 8 ). Although sea otters were twice observed on the surface eating $P$. abrupta in the Barrier Islands (Fig. 1), this species was not a frequent prey for otters even where it was common (Kvitek 1990). Very large pits $(1 \times 2 \mathrm{~m})$, presumably dug by sea otters, and what appeared to be otteropened Panope shells were rarely found at sites where otters and P. abrupta co-occur. Furthermore, at these sites, otters were observed to be consistently feeding on much smaller and more shallowly buried clams (Kvitek 1990). Thus, while T. capax and P. abrupta may offer the highest biomass reward per prey individual, their great burrow depth may either place them out of reach of all but the largest sea otters, or reduce the clam's value as prey to below that of other species due to the high cost of capture (Kvitek et al. 1988). Alternatively, many sea ofters may simply not have learned to identify $P$. abrupta as prey worth the cost of capture. Even though siphons of this species are large and were often conspicuously extended above the sediment surface at sites occupied by otters, until an otter has successfully excavated a $P$. abrupta, it may never know the magnitude of the reward to be gained by digging the huge pit necessary to obtain it. This hypothesis is further supported by the fact that all sea otters currently in southeast Alaska are recent $(20 \mathrm{yr})$ descendents of individuals translocated from the Aleutian Islands and Prince William Sound, areas outside the range of P. abrupta (Morris et al. 1980). Thus, maternal transmission of foraging habits, recently shown to be important in determining otter diet composition (see Riedman \& Estes 1990), may not yet include excavation of $P$. abrupta.

Sea urchins were not only smaller and less abundant at sites occupied by sea otters, but also more cryptic (Table 1). At all but 2 otter sites, urchins could only be found by overturning large rocks along the sampling transects. Otters may also obtain sea urchins by flipping rocks, because at Maurelle Is., where we observed otters foraging on sea urchins most frequently (Kvitek 1990), 56\% of the rocks encountered at the $15 \mathrm{~m}$ sampling depth had been recently overturned. Many of these rocks were surprisingly large (ca $1 \mathrm{~m}$ ). We have also observed this same phenomenon in Prince William Sound (Kvitek unpubl.) and in Washington State (Kvitek et al. 1989).

\section{Importance of butter clams}

Butter clams represent the richest bivalve food source available to sea otters in southeast Alaska. This species accounted for the majority of bivalve biomass at $62 \%$ of the sites sampled throughout southeast Alaska (Tables 3, 4 \& 5) and was larger than other bivalve prey available at most sites (Tables $6 \& 7$ ). The importance of this prey species to sea otters is reflected in the otter-cracked shell record in which butter clams accounted for the majority of shells collected throughout southeast Alaska, and the species was generally more common in the otter-cracked shell record than in the live bivalve assemblage (Table 9). The overrepresentation of butter clams in the predation record may be the result of either sea otter prey preference or the tendency for the shells of the larger butter clams to persist longer than those of smaller species. A preference for butter clams by sea otters would result in the observed shell record pattern, and would be consistent with other studies showing otters to be efficient foragers, preferentially preying on the largest, most calorically valuable prey (Ostfeld 1982, Kvitek et al. 1992). Although prey burrow depth may modify sea otter food preference (see above and Kvitek et al. 1988), all the bivalves encountered in this study, with the exception of Tresus capax and Panope abrupta, were generally found in the top $20 \mathrm{~cm}$ and thus easily excavated by sea otters (Kvitek \& Oliver 1988). Nevertheless, sea otters are known to eat very small clams which are often under-represented in the shell record due to the tendency of sea otters to chew up the entire shell (Kvitek et al. 1992). These smaller clams, however, contribute little to the available biomass compared with the larger butter clams and, thus, may be relatively unimportant as prey (Kvitek et al. 1992).

Although sea otters are commonly characterized as sea urchin predators throughout most of their range (for review see VanBlaricom \& Estes 1988), bivalves in general and butter clams in particular may contribute more to the long-term diet of sea otters in many areas. While sea otters greatly reduce both urchin and clam populations, clams were consistently larger and much more abundant than urchins in areas occupied by sea otters (Tables 1, 3, 4, 6 \& 7). This same pattern was documented in Kodiak (Kvitek et al. 1992). Furthermore, direct observations of feeding otters throughout southeast Alaska showed clams to be the predominant prey (Kvitek 1990). These results suggest that Alaskan sea otters invading a new area initially forage on and rapidly deplete sea urchin populations, but that clams, although significantly reduced in size and abundance by otters, continue to serve as a major prey base long after sea urchins have been virtually eliminated. 
Similar patterns in sea otter prey persistence have been reported from central California for deepburrowing clams (Hines \& Louglin 1980) and abalone in rocky crevices (Hines \& Pearse 1982).

\section{Butter clams, sea otters and paralytic shellifish poisoning}

Because sequestered PST mediates predation on butter clams by sea otters, retained PST may protect some butter clam populations from sea otter predation and may in fact limit the distribution of sea otters, particularly in southeast Alaska where many butter clam populations are chronically toxic (Kvitek et al. 1991). The strength of this argument hinges on the relative importance of butter clams as sea otter prey in southeast Alaska. In the work presented here, we have shown that butter clams make up one of the richest prey resources available to sea otters in southeast Alaska, and based on the otter-cracked shell record, they are the bivalve contributing most to the sea otter diet. The primacy of clams in the diets of southeast Alaskan sea otters is also supported by direct observations of feeding otters (Kvitek 1990). Therefore, while alternative prey may be available throughout the region (e.g. sea urchins and seasonally toxic suspension-feeding bivalves), the majority of available prey biomass at many soft-bottom sites (butter clams) may be unavailable to sea otters in areas subject to toxic dinoflagellate blooms (e.g. the inside passage of southeast Alaska). Furthermore, given the response of other species to ingested toxins producing symptoms similar to those of PST, sea otters may shun areas where they have become sick from PST (Kvitek et al. 1991).

Interestingly, the patterns of otter predation at 2 sites with butter clam toxicity at or above the estimated tolerance threshold for sea otters (148 $\mu \mathrm{g}$ saxitoxin (STX) $100 \mathrm{~g}^{-1}$ clam tissue; Kvitek et al. 1991) support the hypothesis of PST-mediated otter distribution. During the 1987 census, it was found that sea otters in Icy Strait had moved as far east as the western edge of Mud Bay (Fig. 1) (Pitcher 1987). In March 1988 we found abundant and very recent (flesh still attached) otter-cracked shell of butter clams littering the sea floor inside Mud Bay, but no sea otters. Toxicity of the butter clams at the time was $149 \mu \mathrm{g}$ STX $100 \mathrm{~g}^{-1}$ Upon revisiting the site in August 1990, no evidence of subsequent otter foraging was found (e.g. recent or additional ottercracked shells, otter foraging pits, or a decrease in the size or abundance of bivalve prey) in spite of the relatively abundant prey, including green sea urchins (though small) (Tables $1 \& 3$ ). A similar pattern was found in Lisianski Inlet, where only 2 sea otters were observed during the 1987 census (Pitcher 1987).
Although sea otters have been in great abundance at both entrances to the inlet for $>10 \mathrm{yr}$, very few otters have been observed in the inlet (Pitcher 1987; Gail Corbin, Magistrate, Pelican, Alaska, pers. comm.) in spite of the extraordinarily rich clam populations found there (Table 3). The scarcity of otters may be due to the toxicity of butter clams (the dominant bivalve; Table 3 ) which was $>148 \mu \mathrm{g}$ STX $100 \mathrm{~g}^{-1}$ at 2 of 3 sites sampled $\left(72,168\right.$ and $175 \mu \mathrm{g} \mathrm{STX} 100 \mathrm{~g}^{-1}$; State of Alaska Department of Environmental Conservation, Palmer, Alaska, unpubl. data) in the inlet when we were there in March 1990. Sea otters, although abundant in the immediate areas, may be avoiding both Mud Bay and Lisianski Inlet as a result of experiences with PST.

In conclusion, it is unlikely that the rich bivalve populations characteristic of habitats outside the current range of sea otters in southeast Alaska could persist in the presence of sea otters. These populations may, however, be protected from otter predation by PST and the frequent occurrence of toxic dinoflagellate blooms, also characteristic of the region. Furthermore, even nontoxic clam populations within the sea otter range appear to persist longer and provide a more sustainable prey base than the more vulnerable sea urchins.

Acknowledgements. For their invaluable assistance we thank C. Armstrong, S. Baldridge, E. Bowlby, D. Carney, C. Conlin, W. Cunningham, P. Dayton, A. DeGange, B. Hughes, K. Kiese, S. Kim, J. King, H. Lenihan, C. McFadden, J. Oakden, T Oakey, M. Silberstein, P. Slattery, M. Steadler, $J$ Straley, I. Tamlen, and M. Titus. We are also indebted to Captain T. Callahan, and the crew of the RV 'Alpha Helix', as well as C. Allison, M. Ostaz and staff at the Palmer, Alaska Environmental Conservation Shellfish Lab for shellfish toxicity data. Primary support was provided through National Science Foundation grants to J. Oliver (DPP-8619394). Additional support came from National Audubon Graduate Student Research and American Museum of Natural History Lerner-Gray Fund for Marine Research awards to R. Kvitek, and University of Washington Graduate School Research Fund and National Science Foundation awards to R. T Paine.

\section{LITERATURE CITED}

Dayton, P. K. (1975). Experimental studies of algal camopy interactions in a sea otter-dominated kelp community at Amchitka Island, Alaska. Fish. Bull. U.S. 73: 230-237

Duggins, D. (1980). Kelp beds and sea otters: an experimental approach. Ecology $61: 447-453$

Duggins, D. O., Simenstad, S. A., Estes, J. A. (1989). Magnification of secondary production by kelp detritus in coastal marine ecosystems. Science 245: 170-173

Estes, J A., Palmisano, J. F (1974). Sea otters: their role in structuring near shore communities. Science 185: $1058-1060$

Estes, J. A., Smith, N. S., Palmisano, J F. (1978). Sea otter predation and community organization in the western Aleutian Islands, Alaska. Ecology 59: 822-833 
Foster, M. S., Schiel, D. R. (1988). Kelp communities and sea otters: keystone species or just another brick in the wall? In: VanBlaricom, G. R., Estes, J. A. (eds.) The community ecology of sea otters. Springer-Verlag, Berlin, p. 92-115

Hines, A. H., Loughlin, T. R. (1980). Observations of sea otters digging for clams at Monterey harbor, California. NOAA Fish. Bull. 78: 159-163

Hines, H. A., Pearse, J S. (1982). Abalones, shells, and sea otters: dynamics of prey populations in central California. Ecology 63: 1547-1560

Jameson, J. R., Kenyon, K. W., Johnson, A. M., Wight, H. M. (1982). History and status of translocated sea otter populations in North America. Wildl. Soc. Bull. 10: 100-107

Kenyon, C. W. (1969). The sea otter in the eastern Pacific Ocean. N. Am. Fauna 68: 1-352

Kvitek, R. G. (1990). Sea otters and paralytic shellfish poisoning toxins in soft-bottom communities: influence and interactions. Ph.D. dissertation. University of Washington, Seattle

Kvitek R. G. (1991). Sequestered paralytic shellfish poisoning toxins mediate Glaucous-winged gull predation on bivalve prey. Auk 108: 381-392

Kvitek, R. G. (1992). Paralytic shellfish toxins sequestered by bivalves as a defense against siphon nipping fish. Mar Biol. 111: 369-374

Kvitek, R. G., Beitler, M. K. (1991). Relative insensitivity of butter clam neurons to saxitoxin: a pre-adaptation for sequestering paralytic shellish poisoning toxins as a chemical defense. Mar. Ecol. Prog. Ser. 69: 47-54

Kvitek, R. G., DeGange, A. R., Beitler, M. K. (1991) Paralytic shellfish poisoning toxins mediate sea otter feeding behavior. Limnol. Oceanogr. 36: 393-404

Kvitek, R. G., Fukuyama, A. K., Anderson, B. S., Grimm, B. K. (1988). Sea otter foraging on deep-burrowing bivalves in a California coastal lagoon. Mar. Biol. 98: 157-167

Kvitek, R. G., Oliver, J. S. (1988). Sea otter foraging habits and effects on prey populations and communities in softbottom environments. In: VanBlaricom, G. R., Estes, J. A. (eds.) The community ecology of sea otters. SpringerVerlag, Berlin, p. 22-45

Kvitek, R. G., Oliver, J. S., DeGange, A. R., Anderson, B. S. (1992). Changes in Alaskan soft-bottom prey communities

This article was presented by J. S. Pearse, Santa Cruz, California, USA along a gradient in sea otter predation. Ecology 73: $413-428$

Kvitek, R. G., Shull, D., Canestro, D., Bowlby, E. C., Troutman, B. L. (1989). Sea otters and benthic prey communities in Washıngton State. Mar. Mammal. Sci. 5: 266-280

Levin, S. A. (1988) Sea otters and nearshore benthic communuties: a theoretical perspective. In: VanBlaricom, G. R., Estes, J. A. (eds.) The community ecology of sea otters. Springer-Verlag, Berlin, p. 202-209

Miller, D. J., Hardwick, J. E., Dahlstrom, W. A. (1975). Pismo clams and sea otters. Calif. Dep. Fish Game, Mar Res. Tech. Rep. 31 (Sacramento)

Morris R. H., Abbott, D. P., Haderlie, E. C. (1980). Intertidal invertebrates of California. Stanford University Press, Stanford

Nishitani, L., Chew, K. (1988). PSP toxins in the Pacific coast states: monitoring programs and effects on bivalve industries. J. Shellfish Res. 7: 653-669

Ostfeld, R. S. (1982). Foraging strategies and prey switching in the California sea otter. Oecologia 53: 170-178

Paine, R. T. (1969). A note on trophic complexity and community stability. Am. Nat. 103: 91-93

Pitcher, K. W. (1987). Studies of southeastern Alaska sea otter populations: distribution, abundance, structure, range expansion, and potential conflicts with shellfisheries, U.S. Fish and Wildlife Service Cooperative Agreement NO. 1416-0009-954 interim report, Alaska Dep. Fish Game, Anchorage, Alaska

Riedman, M. L., Estes, J. A. (1990). The sea otter (Enhydra lutris): behavior, ecology, and natural history. U.S. Fish Wildl. Serv. Biol. Rep. 90: 0-126

Simenstad, C. A., Estes, J. A., Kenyon, K. W. (1978). Aleuts, sea otters, and alternate stable-state communities. Science 200: 403-411

Stephenson, M. D. (1977). Sea otter predation on pismo clams in Monterey Bay. Calif. Fish Game 63: 117-120

VanBlaricom, G. R., Estes, J. A. (eds.) (1988). The community ecology of sea otters. Springer-Verlag, Berlin

Wendell, F. E., Hardy, R. A., Ames, J. A., Burge, R. T (1986) Temporal and spatial patterns in sea otter (Enhydra lutris) range expansion and in the loss of the pismo clam fisheries. Calif. Fish Game 72: 197-212

Manuscript first received: June 19, 1991

Revised version accepted: February 28, 1992 